\title{
Minimum Fuel Rocket Maneuvers in Horizontal Flight
}

\author{
N. X. VINH* \\ The University of Michigan, Ann Arbor, Mich.
}

\begin{abstract}
This paper presents the complete solution for minimum fuel, lateral turns in a horizontal plane of a rocket powered vehicle. The aerodynamic characteristics of the vehicle are represented by a generalized drag polar. The constraint on the angle-of-attack results in a bound for the bank angle. This upper bound is a function of the weight and of the velocity of the vehicle. The optimal controls in lift, bank, and thrust are expressed explicitely in terms of the state variables. Furthermore the set of adjoint equations in the variational formulation is completely integrated allowing a rigorous discussion of the switching sequences.
\end{abstract}

\section{Nomenclature}

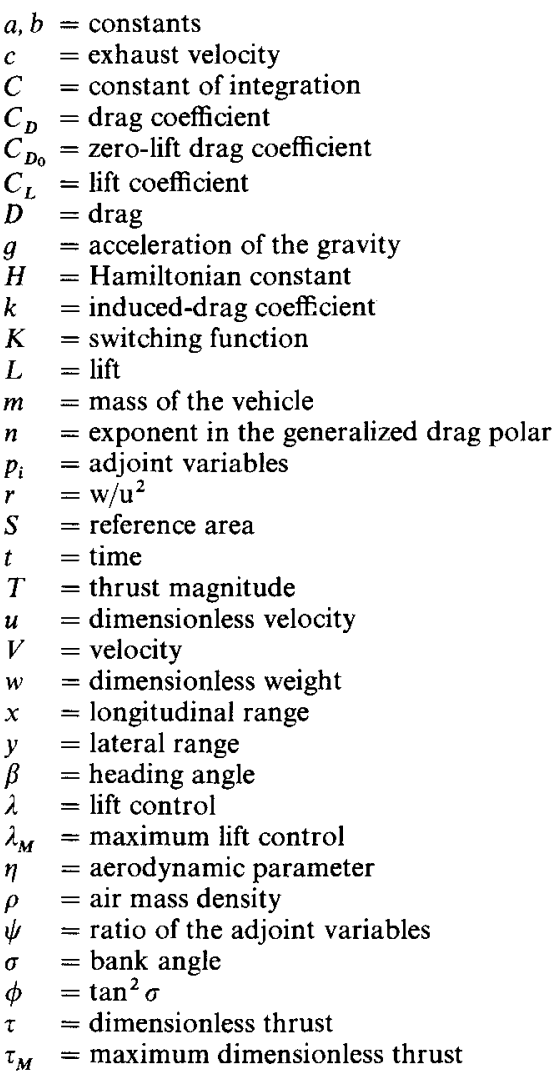

\section{Introduction}

$\mathbf{T}$ HIS paper presents the complete solution for minimum fuel lateral turns in a horizontal plane of a rocket powered vehicle. In Ref. 1 , Connor considers the singular arc portion of this problem while in Ref. 2 , Bryson and Lele give the control laws for the bank angle and the thrust program for the three types of optimal arcs involved. In both papers a parabolic drag polar with unconstrained lift coefficient has been used, and the set of adjoint equations has not been integrated.

In this paper we consider a generalized drag polar. Moreover, it will be shown that, since the angle-of-attack is generally con-

Received April 17, 1972. Work supported by Air Force Grant AFOSR-71-2129.

Index categories: Launch Vehicle and Missile Trajectories; Navigation, Control, and Guidance Theory.

* Professor, Department of Aerospace Engineering. Member AIAA strained, for the vehicle to stay in horizontal flight, the bank angle is bounded by a value, function of the state variables. The optimal thrust profile will be expressed explicitly in terms of the flight velocity. Furthermore, the set of adjoint equations will be completely integrated allowing a rigorous discussion of the optimal switchings from one flight regime to another.

\section{Equations of Flight in a Horizontal Plane}

The equations of motion for a coordinated turn in a horizontal plane, with the thrust always aligned with the velocity, are (Fig. 1)

$$
\begin{gathered}
d x / d t=V \cos \beta, \quad d y / d t=V \sin \beta, \quad d V / d t=(T-D) / m \\
V d \beta / d t=L \sin \sigma / m \\
d m / d t=-T / c
\end{gathered}
$$

For a coordinated turn at constant altitude, we have the constraining relation

$$
L \cos \sigma=m g
$$

We shall use a generalized drag polar of the form

$$
C_{D}=C_{D_{0}}+k C_{L}^{n}
$$

where the zero-lift drag coefficient $C_{D_{0}}$, the induced drag factor $k$, and the exponent $n$ are assumed independent of the Mach number and the Reynolds number. For thin-winged configurations operating in the hypervelocity domain, $n$ is close to $\frac{3}{2}$. We shall use the usual assumption for the lift and drag forces of the form

$$
L=\frac{1}{2} \rho S C_{L} V^{2}, \quad D=\frac{1}{2} \rho S C_{D} V^{2}
$$

Since $x$ and $y$ are ignorable coordinates, if the longitudinal and the lateral ranges are free, we need only consider the equations in $V, \beta$ and $m$. By using $\beta$ as the new independent variable we have

$$
\begin{aligned}
& d V / d \beta=V(T-D) / L \sin \sigma \\
& d m / d \beta=-m V T / c L \sin \sigma
\end{aligned}
$$

We introduce the nondimensional quantities

$$
\begin{gathered}
u=\frac{V}{c}, \quad w=\frac{m g}{\frac{1}{2} \rho S c^{2}\left(C_{D_{0}} / k\right)^{1 / n}}, \quad \eta=\left(k C_{D_{0}}{ }^{n-1}\right)^{1 / n} \\
\tau=T / \frac{1}{2} \rho S c^{2} C_{D_{0}}
\end{gathered}
$$

Fig. 1 Flight in a horizontal plane.

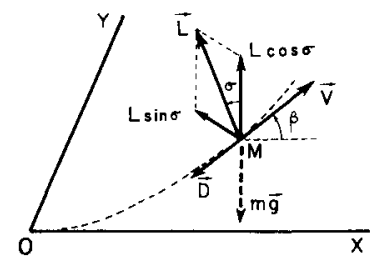


If the angle-of-attack, or equivalently the lift coefficient $C_{L}$, is not constrained, a natural choice for the aerodynamic control would be the bank angle $\sigma$. Then, the lift and the drag coefficients will be obtained from Eqs. (2) and (3). In the practical case where the lift coefficient is bounded, the corresponding bound on the bank angle is function of the state variables $m$ and $V$ through the relation (2). Hence, the lift coefficient will be a better choice as control parameter in this case.

We define a lift control parameter $\lambda$ such that $\lambda^{n}$ is the ratio of the induced drag to the zero-lift drag

$$
\lambda^{n}=\left(k / C_{D_{0}}\right) C_{L}^{n}
$$

then we have

$$
\begin{aligned}
C_{L} & =\left(C_{D_{0}} / k\right)^{1 / n} \lambda, \quad C_{D}=C_{D_{0}}\left(1+\lambda^{n}\right) \\
\cos \sigma & =w / u^{2} \lambda, \quad \sin \sigma=\left(1-w^{2} / u^{4} \lambda^{2}\right)^{1 / 2}
\end{aligned}
$$

The state equations become, with the nondimensional variables

$$
\begin{gathered}
d u / d \beta=\left[\eta u /\left(\lambda^{2} u^{4}-w^{2}\right)^{1 / 2}\right]\left[\tau-\left(1+\lambda^{n}\right) u^{2}\right] \\
d w / d \beta=-\left[\eta u w \tau /\left(\lambda^{2} u^{4}-w^{2}\right)^{1 / 2}\right]
\end{gathered}
$$

where the control variables are the dimensionless thrust $\tau$ and the lift parameter $\lambda$. They are subject to the constraints

$$
0<\tau<\tau_{M}, \quad|\lambda|<\lambda_{M}
$$

To write the variational equations for optimal trajectories, we introduce the adjoint components $p_{1}$ and $p_{2}$ to form the Hamiltonian

$$
\begin{gathered}
H=\left[\eta u \tau /\left(\lambda^{2} u^{4}-w^{2}\right)^{1 / 2}\right]\left(p_{1}-p_{2} w\right)- \\
\eta p_{1}\left(1+\lambda^{n}\right) u^{3} /\left(\lambda^{2} u^{4}-w^{2}\right)^{1 / 2}
\end{gathered}
$$

These adjoint variables are defined by the equations

$$
\begin{aligned}
& \frac{d p_{1}}{d \beta}=\frac{\eta\left(\lambda^{2} u^{4}+w^{2}\right)}{\left(\lambda^{2} u^{4}-w^{2}\right)^{3 / 2}}\left(p_{1}-p_{2} w\right) \tau+\frac{\eta\left(1+\lambda^{n}\right) u^{2}\left(\lambda^{2} u^{4}-3 w^{2}\right)}{\left(\lambda^{2} u^{4}-w^{2}\right)^{3 / 2}} p_{1} \\
& \frac{d p_{2}}{d \beta}=-\frac{\eta u\left(p_{1} w-p_{2} \lambda^{2} u^{4}\right)}{\left(\lambda^{2} u^{4}-w^{2}\right)^{3 / 2}} \tau+\frac{\eta\left(1+\lambda^{n}\right) w u^{3}}{\left(\lambda^{2} u^{4}-w^{2}\right)^{3 / 2}} p_{1}
\end{aligned}
$$

For a minimum fuel trajectory, we maximize the final weight. The solution to the problem is obtained by integrating the systems of state equations (9) and adjoint equations (12) with the appropriate end-conditions, while selecting $\tau$ and $\lambda$, subjected to the constraints [Eq. (10)], in such a way that, at each instant, the Hamiltonian $H$ given by Eq. (11) is an absolute minimum.

\section{Optimal Controls}

First, since $H$ does not contain the independent variable $\beta$, we have the first integral

$$
H=\text { const }
$$

We define the switching function

$$
K=p_{1}-p_{2} w
$$

From consideration of the Hamiltonian (11) we deduce the thrusting law. If

$$
\begin{aligned}
& K<0, \text { we select } \tau=\tau_{M} \quad \text { (boosting phase) } \\
& K>0, \text { we select } \tau=0 \quad \text { (coasting phase) } \\
& K=0 \text { for a finite time interval } \\
& \text { we selected } \tau=\text { variable (sustaining phase) }
\end{aligned}
$$

Considered as function of $\lambda, H$ reaches a minimum either at

$$
\lambda=\lambda_{M}
$$

or at an interior point given by $\partial H / \partial \lambda=0$. Explicitly we have

$$
\left(p_{1}-p_{2} w\right) \tau=u^{2} p_{1}\left[1-(n-1) \lambda^{n}+n w^{2} u^{-4} \lambda^{n-2}\right]
$$

Along a coasting arc, $\tau=0$, and along a sustaining arc, $K=0$. In both cases the equation is simplified to give an explicit law for the lift control

$$
\left[(n-1) \lambda^{n}-1\right] / n \lambda^{n-2}=w^{2} / u^{4}
$$

In terms of the bank angle

$$
\left[(n-1)-n \cos ^{2} \sigma\right] / \cos ^{n} \sigma=u^{2 n} / w^{n}
$$

We shall refer to the interior lift and bank as the normal lift and the normal bank controls. Along a boosting arc, the optimal lift control is either the maximum lift or as given by Eq. (17) with $\tau=\tau_{M}$. In this case, $\lambda$ is also function of $p_{1}$ and $p_{2}$.

Along a sustaining arc, relation (18) can be further simplified. In general, let

$$
\psi=p_{2} / p_{1}
$$

Then

$$
d \psi / d \beta=-\left(\psi / p_{1}\right) d p_{1} / d \beta+\left(1 / p_{1}\right) d p_{2} / d \beta
$$

Using Eqs. (12) and (20) and rearranging, we have a Riccati equation for $\psi$

$$
\begin{aligned}
& d \frac{\eta \psi}{d \beta}-\frac{\eta w\left(\lambda^{2} u^{4}+w^{2}\right)}{\left(\lambda^{2} u^{4}-w^{2}\right)^{3 / 2}} \tau \psi^{2}+\frac{\eta}{\left(\lambda^{2} u^{4}-w^{2}\right)^{3 / 2}} \times \\
& {\left[\left(\lambda^{2} u^{4}-\lambda^{2} u^{5}+w^{2}\right) \tau+\left(1+\lambda^{n}\right) u^{2}\left(\lambda^{2} u^{4}-3 w^{2}\right)\right] \psi+} \\
& \frac{\eta u w}{\left(\lambda^{2} u^{4}-w^{2}\right)^{3 / 2}}\left[\tau-\left(1+\lambda^{n}\right) u^{2}\right]=0
\end{aligned}
$$

Explicit solution for this equation can be obtained for the case of sustaining flight and for the case of coasting flight.

Along a sustaining $\operatorname{arc}, K=0$, and we have

$$
\psi=1 / w
$$

By substituting this solution into Eq. (21) and using Eq. (9) we have

$$
\lambda^{2}=w^{2}(3+u) / u^{4}
$$

Relations (22) and (23) characterize the sustaining flight regime for a finite time interval, and they are valid for both the normal lift and maximum lift control. For normal lift, using the relation (8) for the bank angle, we deduce the simple law for banking along a sustaining arc

$$
\cos ^{2} \sigma=1 /(3+u)
$$

We notice that, it is remarkable that this optimal bank angle is independent of the numerical coefficients in the generalized drag polar [Eq. (3)]. The lift control can also be expressed explicitly in terms of the dimensionless velocity. Combining Eqs. (18) and (23) we have

$$
\lambda^{n}=(3+u) /[(n-1) u+(2 n-3)]
$$

Finally, upon elimination of $\lambda$ between Eqs. (18) and (23) we have the relation between the dimensionless weight and velocity along a sustaining arc, using normal lift

$$
w^{n} u^{-2 n}(3+u)^{(n / 2)-1}[(n-1) u+(2 n-3)]=1
$$

By taking the derivative of this equation with respect to $\beta$, and substituting the appropriate derivatives from the state equations (9), we have, after rearranging, the thrust magnitude profile along a sustaining arc with normal lift control

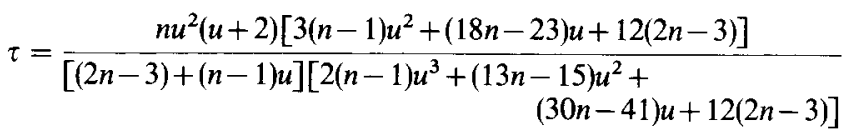

where $n=2$ we have

$$
\tau=\frac{2 u^{2}(2+u)(4+3 u)}{(1+u)\left(4+5 u+2 u^{2}\right)}
$$

For sustaining flight, using maximum lift, we write Eq. (23) as

$$
w=\lambda_{M} u^{2} /(3+u)^{1 / 2}
$$

This relation replaces (26) when maximum lift is optimal. By taking its derivative and using the state equations (9) we have the thrust magnitude profile along a sustaining arc with maximum lift control

$$
\tau=3\left(1+\lambda_{M}{ }^{n}\right)(4+u) u^{2} /\left(2 u^{2}+9 u+12\right)
$$

We notice that the bank angle is also given by Eq. (24). Explicit formula for the normal lift along a maximum thrust arc is not known. For the case where the flight is at maximum lift, by dividing the state equations (9) and using $\lambda=\lambda_{M}, \tau=\tau_{M}$ we have

$$
d u / d w=-(1 / w)\left(1-a u^{2}\right)
$$

where 
Table 1 Optimal controls

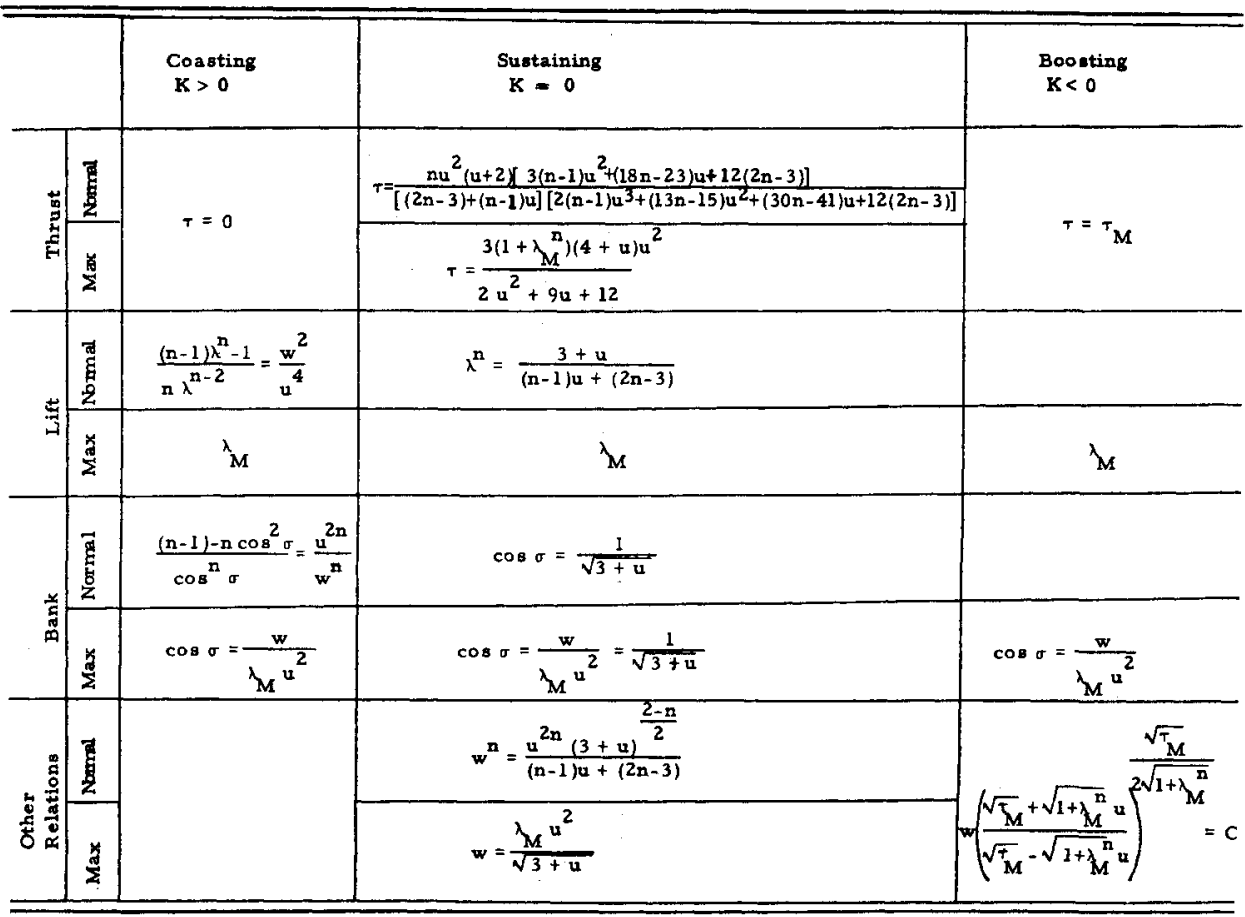

By integrating we have

$$
a=\left(1+\hat{\lambda}_{M}{ }^{n}\right) / \tau_{M}
$$

$$
w\left(\frac{1+(a)^{1 / 2} u}{1-(a)^{1 / 2} u}\right)^{1 /[2(a) 1 / 2]}=C
$$

where $C$ is a const.

When $\tau_{M} \rightarrow \infty, a \rightarrow 0$, we write $b=1 / 2(a)^{1 / 2}$ and consider

$$
\left(\frac{1+(a)^{1 / 2} u}{1-(a)^{1 / 2} u}\right)^{1 /\left[2(a)^{1 / 2}\right]}=\left[\left(1+\frac{u}{b}\right)+0\left(\frac{1}{b^{2}}\right)\right]^{b} \rightarrow e^{u} \text { as } b \rightarrow \infty
$$

Hence, if impulsive thrust is permitted, we have, along an impulsive thrust arc

$$
w e^{u}=C
$$

This relation can be obtained by integrating Eq. (31) with $a=0$.

In the minimum fuel turning to a specific heading angle, if pure coasting flight leads to a final velocity higher or equal to the prescribed final velocity, the fuel consumption is zero. Hence subsequently we shall assume that the prescribed final condition is such that the trajectory shall include at least one thrusting arc. The results of this section are collected in Table 1.

\section{Optimal Switchings}

Both the lift (and hence the bank) and the thrust controls can switch from one mode to another. For the normal bank angle, we see from Eqs. (19) and (24) that $\sigma$ varies between $\arccos [(n-1) / n]^{1 / 2}$ and $90^{\circ}$ for coasting flight and between $54^{\circ} 44^{\prime}$ and $90^{\circ}$ for sustaining flight, hence at a rather high angle. Therefore, if the lift coefficient is constrained, the flight may be effectuated at the maximum permissible bank angle. This angle is a function of the state variables $w$ and $u$ and is given by

$$
\cos \sigma_{\max }=w / \lambda_{M} u^{2}
$$

A convenient graphical discussion of optimal switching in bank is obtained using polar coordinates. Let

$$
r=w / u^{2}
$$

Along a flight at maximum bank angle, we have

$$
r=\lambda_{M} \cos \sigma
$$

The plot of this relation is a circle (Fig. 2). On the other hand, except for boosting flight at $\tau=\tau_{M}$, the normal optimal bank angle is given by Eq. (19) which can be written as

$$
r^{n}=\cos ^{n} \sigma /\left[(n-1)-n \cos ^{2} \sigma\right]
$$

The plot representing this relation starts at the origin for $\sigma=90^{\circ}$ and tends asymptotically to $r=\infty$ when $\sigma=\arccos [(n-1) / n]^{1,2}$. It should be noted that, for horizontal flight, $r$ is bounded by $r \leqq \lambda_{M}$. The two curves intersect each other at the point

$\sigma^{*}=\arccos \left[\frac{(n-1) \lambda_{M}{ }^{n}-1}{n \lambda_{M}{ }^{n}}\right]^{1 / 2}, \quad r^{*}=\left[\frac{(n-1) \lambda_{M}{ }^{n}-1}{n \lambda_{M}{ }^{n-2}}\right]^{1 / 2}$

The point is real when

$$
\lambda_{M}{ }^{n}>1 /(n-1)
$$

Hence, if this condition is satisfied, the flight is at normal bank angle whenever

$$
\frac{w}{u^{2}} \leqq\left[\frac{(n-1) \lambda_{M}{ }^{n}-1}{n \lambda_{M}{ }^{n-2}}\right]^{1 / 2}
$$

It is at maximum bank angle for

$$
\left[\frac{(n-1) \lambda_{M}{ }^{n}-1}{n \lambda_{M}{ }^{n-2}}\right]^{1 / 2}<\frac{w}{u^{2}} \leqq \lambda_{M}
$$

From Eq. (39) we see that, for a given aerodynamic configuration of the vehicle, the switching is always made at a fixed bank angle. Along a coasting arc, the switching in lift and bank is made at different values of $w$ and $u$ but in such a way that the ratio $w / u^{2}$ is constant. On the other hand, if the switching is made along a sustaining arc, because of the additional relation

Fig. 2 Switching in bank angle.

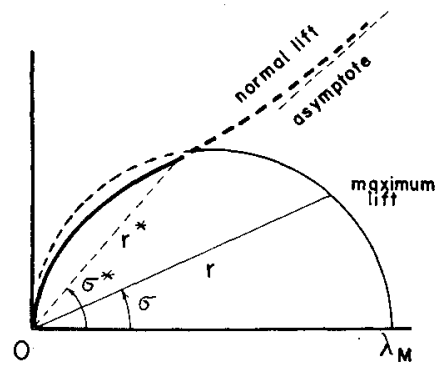


(23), it is always at a prescribed weight and a prescribed velocity. These are given by

$$
\begin{aligned}
w^{*} & =\frac{1}{n^{1 / 2} \lambda_{M}{ }^{(n-2) / 2}} \frac{\left[(3-2 n) \lambda_{M}{ }^{n}+3\right]^{2}}{\left[(n-1) \lambda_{M}{ }^{n}-1\right]^{3 / 2}} \\
u^{*} & =\frac{(3-2 n) \lambda_{M}{ }^{n}+3}{(n-1) \hat{\lambda}_{M}{ }^{n}-1}
\end{aligned}
$$

The switching velocity $u^{*}$ is positive when

$$
1 /(n-1)<\lambda_{M}{ }^{n}<3 /(2 n-3)
$$

For a vehicle with low lifting capability, condition (40) is usually violated and the trajectory is entirely flown at $\lambda_{M}$ and at the maximum permissible bank angle as given by Eq. (35).

The switching for the thrust program is governed by the function $\psi$. From Eq. (11), along a coasting arc, for a minimizing Hamiltonian $p_{1}$ is negative and since $K=p_{1}-p_{2} w>0$ along this arc, we can write the thrusting condition (15)

$$
\begin{array}{ll}
\psi>1 / w & \text { (coasting phase) } \\
\psi \leqq 1 / w & \text { (boosting phase) } \\
\psi=1 / w & \text { (sustaining phase) }
\end{array}
$$

Hence the engine is activated as soon as $\psi=1 / w$. The function $\psi$ is given by the Riccati equation (21). Along a coasting arc, $\tau=0$ and the equation becomes linear. Using $u$ as the new independent variable, we have

$$
\frac{d \psi}{d u}-\frac{\left(\lambda^{2} u^{4}-3 w^{2}\right)}{u\left(\lambda^{2} u^{4}-w^{2}\right)} \psi+\frac{w}{\left(\lambda^{2} u^{4}-w^{2}\right)}=0
$$

We notice that $w$ is a constant along a coasting arc. If the flight is at $\lambda=\lambda_{M}$ the solution of this equation is

$$
\psi=C u^{3} /\left(\lambda_{M}{ }^{2} u^{4}-w^{2}\right)^{1 / 2}-u / 2 w
$$

where $C$ is a constant of integration. For flight at normal lift, for a parabolic drag polar $n=2$, and $\lambda^{2}$ can be easily expressed in terms of $u$ through the Eq. (18). For arbitrary $n$ we use the new variable

$$
\phi=\tan ^{2} \sigma
$$

and rewrite Eq. (19)

$$
u^{2 n} / w^{n}=[(n-1) \phi-1](\phi+1)^{(n-2) / 2}
$$

Hence

$$
d \phi / d u=4(\phi+1)[(n-1) \phi-1] / u[(n-1) \phi+1]
$$

And the equation for $\psi$ along a coasting arc at normal lift becomes

$$
\frac{d \psi}{d \phi}-\frac{(\phi-2)[(n-1) \phi+1]}{4 \phi(\phi+1)[(n-1) \phi-1]} \psi+\frac{u[(n-1) \phi+1]}{4 w \phi(\phi+1)[(n-1) \phi-1]}=0
$$

In terms of the bank angle, the solution of this equation is $\psi=C \phi^{1 / 2}(\phi+1)^{3(n-2) / 4 n}[(n-1) \phi-1]^{(3-2 n) / 2 n}-$

$$
\frac{(\phi+1)^{(n-2) / 4 n}}{2 w^{1 / 2}}[(n-1) \phi-1]^{1 / 2 n}
$$

where $C$ is the constant of integration. When $n=2$, $\phi=1+u^{4} w^{-2}$ and we have

$$
\psi=(C / u)\left(u^{4} / w^{2}+1\right)^{1 / 2}-u / 2 w
$$

where one constant term in $w$ has been included in the new constant $C$. The expression (47) for $\psi$ along a coasting arc at $\lambda_{M}$, and (52) for $\psi$ along a coasting are using normal lift, can be used to prove rigorously the existence of the six types of optimal trajectories as has been displayed by Bryson and Lele. ${ }^{2}$ For ease of notation we shall use the expression (53) for normal flight using a parabolic drag polar. The conclusions are the same if expression (47) or (52) is used instead. With the impulsive approximation for the boosting arc, we have the following theorems.

Proposition 1: there is no coasting arc between two thrusting arcs.

Consider a coasting arc between two thrusting arcs. Along the coasting arc, the expression for $\psi$ is given by Eq. (53). Let $u_{1}$ and $u_{2}$ be the velocities at the two ends of this arc, with $u_{1}>u_{2}$, and let the constant weight be $w_{1}=w_{2}=w$. We can calculate the constant of integration $C$ at the point 1 . At this point which is the end of a thrusting phase, $\psi=1 / w$. Hence

$$
1 / w=\left(C / u_{1}\right)\left(u_{1}{ }^{4} / w^{2}+1\right)^{1 / 2}-u_{1} / 2 w
$$

this gives

$$
C=\left(u_{1} / w\right)\left(1+u_{1} / 2\right)\left(u_{1}^{4} / w^{2}+1\right)^{-1 / 2}
$$

We have similar relation with $u_{1}$ replaced by $u_{2}$ if we evaluate the constant of integration $C$ at the point 2 . Therefore we have the relation between $u_{1}$ and $u_{2}$

$u_{1}\left(1+u_{1} / 2\right)\left(u_{1}^{4} / w^{2}+1\right)^{-1 / 2}=u_{2}\left(1+u_{2} / 2\right)\left(u_{2}{ }^{4} / w^{2}+1\right)^{-1 / 2}$

Now consider the function

$$
f(u)=u(1+u / 2)\left(u^{4} / w^{2}+1\right)^{-1 / 2}
$$

Since $u_{1} \neq u_{2}$, a necessary condition for relation (55) to exist is that the function $f(u)$ passes at least through a maximum or a minimum for a certain value $\bar{u} \varepsilon\left[u_{2}, u_{1}\right]$. This value $\bar{u}$ is given by

$$
(1+u)\left(u^{4} / w^{2}+1\right)^{-1 / 2}-\left(2 u^{4} / w^{2}\right)(1+u / 2)\left(u^{4} / w^{2}+1\right)^{-3 / 2}=0
$$
or

$$
w^{2}=\vec{u}^{4} /(1+\hat{u})
$$

This is the same relation as Eq. (26) with $n=2$. Hence the point $(w, \bar{u})$ is on the switching surface for sustaining flight and the arc from 1 to 2 is not a pure coasting arc. This contradiction completes the proof.

Proposition 2: A thrusting arc between two coasting arcs is not possible for impulsive thrust.

At the ends of the thrusting arcs we have

$$
\psi_{1}=1 / w_{1}, \quad \psi_{2}=1 / w_{2}
$$

The function $\psi$ is continuous since it is the ratio of two continuous adjoint variables. If the thrusting arc is an impulsive one, at the point of application of the impulse we have $\psi_{1}=\psi_{2}$. This is not possible since the impulse produces a discontinuity in the weight, that is $w_{1} \neq w_{2}$.

Proposition 3: The initial arc is a coasting arc if

$$
w_{0}<u_{0}^{2} /\left(1+u_{0}\right)^{1 / 2}
$$

It is a boosting arc if

$$
w_{0}>u_{0}^{2} /\left(1+u_{0}\right)^{1 / 2}
$$

Consider the case of an initial coasting arc followed by a thrusting arc. In the $(w, u)$ plane the coasting arc starts at the point $\left(w_{0}, u_{0}\right)$ and ends at the point $\left(w_{0}, u_{1}\right)$ with $u_{0}>u_{1}$. Along th:s arc the function $\psi$ is given by Eq. (53). If we evaluate the constant of integration at the point $\left(w_{0}, u_{1}\right)$, we have along the

\begin{tabular}{|c|c|}
\hline BC & \\
\hline BSC & and \\
\hline & \\
\hline
\end{tabular}
initial coasting arc

$\psi=\left(u_{1} / w_{0} u\right)\left(1+u_{1} / 2\right)\left(u_{1}{ }^{4} / w_{0}{ }^{2}+1\right)^{-1 / 2}\left(u^{4} / w_{0}{ }^{2}+1\right)^{1 / 2}-u / 2 w_{0}$

By Eq. (45), $\psi>1 / w_{0}$ along the coasting arc, and in particular at the initial point $\left(w_{0}, u_{0}\right)$. Hence, at this point we have $u_{1}\left(1+u_{1} / 2\right)\left(u_{1}{ }^{4} / w_{0}{ }^{2}+1\right)^{-1 / 2}>u_{0}\left(1+u_{0} / 2\right)\left(u_{0}{ }^{4} / w_{0}{ }^{2}+1\right)^{-1 / 2}$

Each side of the inequality has the same functional form as $f(u)$ given by Eq. (56). We have shown that this function is stationary along the variable thrust switching surface, and hence Eq. (61) shows that the function is decreasing at $\left(w_{0}, u_{0}\right)$. Mathematically, we have

$$
(d f / d u)_{u=u_{0}}<0
$$

By carrying out the operation, we have the condition in Eq. (58). A similar proof shows that when the inequality [Eq. (59)] is satisfied, the initial arc is a boosting arc. Of course, for the very special case where an equality exists, the trajectory starts with a variable thrusting arc.

Let $B=$ Boosting arc, $C=$ Coasting arc and, $S=$ Sustaining arc, we see now that there exist six possible types of optimal trajectories as have been displayed in Ref. 2, namely 
Fig. 3 Phase space and switching surfaces.

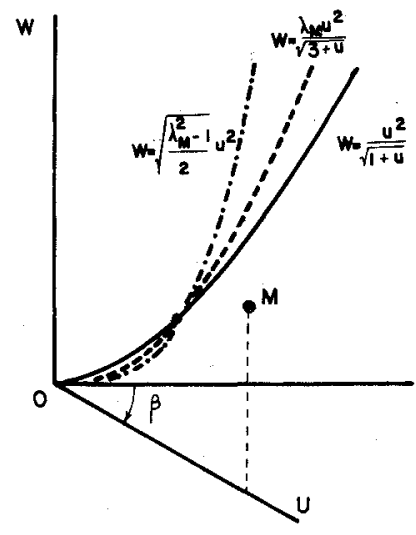

\section{Optimal Trajectories}

The totality of optimal trajectories is contained in the cylindrical coordinates space $(w, u, \beta)$ (Fig. 3). For simplicity we consider a parabolic drag polar. In this space we have the following switching surfaces of revolution:

Sustaining flight using normal lift

$$
w=u^{2} /(1+u)^{1 / 2}
$$

Sustaining flight using maximum lift

$$
w=\lambda_{M} u^{2} /(3+u)^{1 / 2}
$$

Switching from normal lift to maximum lift

$$
w=\left[\left(\lambda_{M}{ }^{2}-1\right) / 2\right]^{1 / 2} u^{2}
$$

When $\lambda_{\max }<1$, the flight is always at maximum lift. The conditions in Eqs. (58) and (59) and similar conditions for the case of maximum-lift flight indicate the initial arc. Subsequent selection of one of the six types of trajectories in Eq. (62) depends on the terminal conditions as discussed in Ref. 2 . In the same reference, the change in heading angle has been given for the case of normal lift. The velocity decreases both along a coasting arc and along a sustaining arc. In the notation of this paper, with $u_{1}>u_{2}$ we have

Along a coasting arc using normal lift, the change in heading is

$$
(\Delta \beta)_{C}=\frac{1}{4 \eta}\left\{\log \left[u^{2}+\left(u^{4}+w^{2}\right)^{1 / 2}\right]\right\}_{u_{2}}^{u_{1}}
$$

Along a sustaining arc with normal lift, we have

$$
\begin{aligned}
&(\Delta \beta)_{S}= \frac{1}{4 \eta}\left[-\frac{2[(u+1)(u+2)]^{1 / 2}}{u}+\right. \\
& 2 \log \left\{2[(u+1)(u+2)]^{1 / 2}+2 u+3\right\}- \\
&\left.(2)^{1 / 2} \log \left(\frac{2(2)^{1 / 2}[(u+1)(u+2)]^{1 / 2}+3 u+4}{u}\right)\right]_{u_{2}}^{u_{1}}
\end{aligned}
$$

For flight at maximum lift, these expressions are replaced by

$$
\begin{array}{r}
(\Delta \beta)_{C}=\frac{1}{2 \eta\left(1+\lambda_{M}{ }^{2}\right)}\left[\lambda_{M} \log \left[\lambda_{M} u^{2}+\left(\lambda_{M}{ }^{2} u^{4}-w^{2}\right)^{1 / 2}\right]-\right. \\
\left.\frac{\left(\lambda_{M}{ }^{2} u^{4}-w^{2}\right)^{1 / 2}}{u^{2}}\right]_{u_{2}}^{u_{1}}
\end{array}
$$

and

$$
\begin{array}{r}
(\Delta \beta)_{S}=\frac{\lambda_{M}}{\eta\left(1+\lambda_{M}^{2}\right)}\left[\log \left\{2[(u+2)(u+3)]^{1 / 2}+2 u+5\right\}-\right. \\
\frac{2}{(6)^{1 / 2}} \log \left(\frac{2[6(u+2)(u+3)]^{1 / 2}+5 u+12}{u}\right)- \\
\left.\frac{(u+2)[(u+2)]^{1 / 2}}{u(u+3)^{1 / 2}}\right]_{u_{2}}^{u_{1}}
\end{array}
$$

\section{References}

${ }^{1}$ Connor, M. A., "Optimization of a Lateral Turn at Constant Height," AIAA Journal, Vol. 5, No. 2, Feb. 1967, pp. 335-338.

${ }^{2}$ Bryson, A. E., Jr. and Lele, M. M., "Minimum Fuel Lateral Turns at Constant Altitude," AI AA Journal, Vol. 7, No. 3, March 1969, pp: $559-560$. 